\title{
CID16020046, a GPR55 antagonist, attenuates sepsis-induced acute kidney injury
}

\author{
RONGXIN CHEN $^{1}$, HAILIN XU $^{1}$, ZEBIN GUO $^{2}$, PENG ZHANG $^{1}$, JIANXIA CHEN $^{2}$ and ZHENG CHEN ${ }^{1}$ \\ ${ }^{1}$ Organ Transplant Center, The Second Affiliated Hospital of Guangzhou Medical University; \\ ${ }^{2}$ Department of Intensive Care Unit, The Second Affiliated Hospital of Guangzhou Medical University, \\ Guangzhou, Guangdong 511447, P.R. China
}

Received November 30, 2020; Accepted March 17, 2021

DOI: $10.3892 / \mathrm{mmr} .2022 .12671$

\begin{abstract}
Acute kidney injury (AKI) is the most common and serious complication of sepsis, and it is also the main cause of mortality in patients with sepsis. The G protein-coupled receptor 55 (GPR55) inhibitor CID16020046 was found to suppress the inflammatory response in sepsis models in mice. The aim of the present study was to investigate the effect of CID16020046 on AKI in sepsis mouse models and elucidate the possible underlying mechanisms. A sepsis model in mice was established by cecal ligation/perforation (CLP). The expression levels of GPR55 in the serum of patients with sepsis and the renal tissues of septic mice were determined via reverse transcription-quantitative PCR and western blot analyses, respectively. The pathological injury of renal tissue was evaluated using $H \& E$ and periodic acid-Schiff staining. ELISA was performed to detect the levels of renal injury-related factors, including blood urea nitrogen (BUN), creatinine (Cre), kidney injury molecule 1 (KIM1) and neutrophil gelatinase-associated lipocalin (NGAL) in septic mice. Moreover, the levels of pro-inflammatory cytokines (TNF- $\alpha$, IL-6 and IL-1 $\beta$ ) were detected via ELISA and western blotting. Apoptosis was determined using TUNEL staining and western blotting. The expression levels of Rho-associated protein kinase (ROCK) pathway-related proteins (Ras homolog family member A, ROCK1 and ROCK2) was measured via western blotting. Finally, H\&E staining was used to evaluate the effect of CID16020046 on various organs in mice. Compared with the control subjects, the expression level of GPR55 in the serum of patients with sepsis was significantly increased. Compared with the sham group, CID16020046 (20 mg/kg) significantly decreased the levels of BUN and Cre in the serum, as well as the contents of KIM1 and NGAL in the urine. Furthermore,
\end{abstract}

Correspondence to: Professor Zheng Chen, Organ Transplant Center, The Second Affiliated Hospital of Guangzhou Medical University, 250 Changgang East Road, Guangzhou, Guangdong 511447, P.R. China E-mail: shangxiaohai123@163.com

Key words: acute kidney injury, sepsis, CID16020046, G proteincoupled receptor 55
CID16020046 significantly decreased the contents of TNF- $\alpha$, IL- 6 and IL-1 $\beta$ in the serum and renal tissue of septic mice, and reduced cell apoptosis. In addition, CID16020046 effectively suppressed the expression levels of ROCK pathway-related proteins, and H\&E staining revealed that CID16020046 $(20 \mathrm{mg} / \mathrm{kg}$ ) had no toxic effect on the heart, liver, spleen or lung in normal mice. In conclusion, CID16020046 may prove useful for the development of drugs for the treatment of sepsis-induced AKI.

\section{Introduction}

Sepsis is a systemic inflammatory response syndrome caused by infection, which is associated with high global morbidity and mortality rates between 1979 and 2015 (1-3). Although the application of antibiotics, vasoactive agents and renal dialysis in the treatment of sepsis has greatly improved the prognosis, the fatality rate of sepsis remains high (4). Therefore, it is crucial to develop novel and effective treatments for sepsis in order to improve the diagnostic accuracy and patient prognosis.

Acute kidney injury (AKI) is a common complication in patients with severe sepsis. According to statistics, 50\% of AKI cases are associated with sepsis and the fatality rate is as high as $40 \%$ (5). It was previously reported that sepsis was characterized by inflammation in the early stage and by apoptosis-related immunosuppression in the late stage (6). Therefore, inflammation and apoptosis are the key mechanisms implicated in terminal organ damage in sepsis.

The $\mathrm{G}$ protein-coupled receptor 55 (GPR55) is a newly discovered $\mathrm{G}$ protein-coupled receptor, which is mainly located in brain, gastrointestinal tract, spleen, endothelial cells and other regions of the body, and is involved in the regulation of a series of physiological activities, including nerve or inflammatory pain, gastrointestinal inflammation, obesity, type 2 diabetes and cancer (7). GPR55 is closely associated with the occurrence and progression of inflammation. For instance, Schicho et al (8) revealed that the expression level of GPR55 in the gastrointestinal epithelial cells of rats treated with lipopolysaccharide was significantly increased, indicating that GPR55 may be involved in intestinal inflammation. In addition, Staton et al (9) reported that GPR55 knockdown mice had a high tolerance to inflammation induced by Freund's complete adjuvant, and the levels of pro-inflammatory factors, such as 
IL-4, were significantly increased in GPR55 knockdown mice. Through the further study of GPR55, it was observed that the GPR55 antagonist CID16020046 had significant anti-inflammatory properties; for example, CID16020046 effectively inhibits intestinal inflammation in mice by reducing myeloperoxidase activity and inflammation (10). Of note, a previous study revealed that inhibition of GPR55 effectively reduced the production of the pro-inflammatory cytokines TNF- $\alpha$ and IL-6 in sepsis models (11). However, to the best of our knowledge, it has not been investigated whether inhibition of GPR55 affects sepsis-induced AKI.

Therefore, the aim of the present study was to systematically and comprehensively investigate the role and potential underlying molecular mechanism of GPR55 inhibitors in a septic mouse model by detecting the levels of related renal injury indicators and inflammatory cytokines.

\section{Materials and methods}

Human serum samples. Peripheral blood $(6 \mathrm{ml})$ was collected from 15 patients with sepsis and 15 healthy controls. The patients included nine men and six women aged 18-55 years old, and the inclusion criteria and the exclusion criteria for patients with sepsis referred to a previous study (12). The samples were collected from the Emergency Department of The Second Affiliated Hospital of Guangzhou Medical University from July 2015 to December 2016. The study protocol was approved by the Ethics Committee of The Second Affiliated Hospital of Guangzhou Medical University. All the subjects who participated in the present study signed the informed consent form.

Reverse transcription-quantitative PCR (RT-qPCR) analysis. Total RNA was extracted from patients' serum samples using TRIzol ${ }^{\circledR}$ reagent (Invitrogen; Thermo Fisher Scientific, Inc.). Subsequently, RNA was reverse-transcribed into cDNA for 15 min at $42^{\circ} \mathrm{C}$ using a PrimeScript RT Reagent kit (Takara Biotechnology Co., Ltd.).qPCR was performed using TB Green Fast qPCR mix (Takara Biotechnology Co., Ltd.) according to the manufacturer's instructions, using cDNA as the template. The primer sequences were as follows: GPR55 forward, 5'-TCT ACATGATCAACCTGGCAGTCT-3' and reverse, 5'-CTG GGACAGGACCATCTTGAA-3'; and GAPDH forward, 5'-ATGGGGAAGGTGAAGGTCG-3' and reverse, 5'-GGG GTCATTGATGGCAACAATA-3'. The reaction conditions were as follows: $50^{\circ} \mathrm{C}$ for $2 \mathrm{~min}$, initial denaturation at $95^{\circ} \mathrm{C}$ for $10 \mathrm{~min}$, followed by 40 cycles of $95^{\circ} \mathrm{C}$ for $15 \mathrm{sec}, 60^{\circ} \mathrm{C}$ for $15 \mathrm{sec}$ and $72^{\circ} \mathrm{C}$ for $15 \mathrm{sec}$. The relative expression of GPR55 was calculated using the $2^{-\Delta \Delta \mathrm{Cq}}$ method (13).

Cecal ligation/perforation (CLP) model and experimental groups. In total, 36 male C57BL/6J mice (weight, 21-25 g; age, 6 weeks) were purchased from the Qinglongshan Laboratory Animal Farm (Nanjing, China). The mice were housed in a standard environment including a 12-h light/dark cycle, controlled temperature $\left(22 \pm 2^{\circ} \mathrm{C}\right)$ and humidity $(55 \pm 5 \%)$ with free access to water and food. The GPR55 inhibitor (CID16020046) was purchased from ChemDiv, Inc. Animals were adaptively fed for 1 week and fasted for $12 \mathrm{~h}$ before the start of the experiment.
The mice were randomly divided into four groups ( $n=9 /$ group) as follows: Control, sham, CLP and CID16020046 $(20 \mathrm{mg} / \mathrm{kg})$ groups. A sepsis model in mice was established via CLP. In brief, the mice were intraperitoneally injected with $50 \mathrm{mg} / \mathrm{kg}$ pentobarbital sodium. After the mice were anesthetized, a 2-cm incision was made in the abdominal wall of the mice using a scalpel under aseptic conditions, the cecum was ligated with no. 3 silk thread and then the cecum was perforated twice with a no. 18 injection needle. Subsequently, the peritoneum and skin were intermittently sutured with no. 4 silk thread. In addition, $50 \mathrm{mg} / \mathrm{kg}$ saline was injected subcutaneously to prevent the development of shock in mice. No cecal ligation was performed in the sham group. The CID16020046 group was injected intraperitoneally with CID16020046 solution $(20 \mathrm{mg} / \mathrm{kg}$ ) immediately following CLP surgery (10), while the other groups were injected with an equal volume of saline. Urine samples were collected from mice in each group $12 \mathrm{~h}$ after the operation. After $24 \mathrm{~h}$, all mice were euthanized by cervical dislocation, and $2 \mathrm{ml}$ blood samples and renal tissue samples were collected for the subsequent experiments. The animal experimental procedures were approved by the Animal Committee of The Second Affiliated Hospital of Guangzhou Medical University.

Histopathological examination. The tissue samples of mice were examined following $\mathrm{H} \& \mathrm{E}$ and periodic acid-Schiff (PAS) staining. For H\&E staining, at $24 \mathrm{~h}$ after dissection, the heart, liver, spleen, lung and kidney tissues of each group were fixed with $4 \%$ formaldehyde for $24 \mathrm{~h}$ at room temperature and embedded in paraffin after dehydration. The tissues were cut into $4-\mu \mathrm{m}$ sections and stained with hematoxylin for $15 \mathrm{~min}$ and eosin for $5 \mathrm{~min}$ both at room temperature, and the histopathological changes of each organ were observed under a light microscope (Leica DM 500; Leica Microsystems GmbH) at a magnification of $\mathrm{x} 400$.

For PAS staining, $1-\mathrm{mm}^{3}$ of kidney tissue was fixed with $10 \%$ formaldehyde $4^{\circ} \mathrm{C}$ for $24 \mathrm{~h}$, dehydrated using an ethanol gradient through $30,50,70,80,95$ and $100 \%$ ethanol successively, embedded in paraffin and cut into $5-\mu \mathrm{m}$ sections. These sections were then stained with PAS at room temperature for 10 min and observed and imaged under a light microscope (magnification, x200).

Renal function assessment. After $24 \mathrm{~h}$ of modeling, the abdominal aortic blood $(2 \mathrm{ml})$ of mice in each group was collected and centrifuged at $3,000 \mathrm{x}$ g for $5 \mathrm{~min}$ at $4^{\circ} \mathrm{C}$. According to the manufacturer's instructions, blood urea nitrogen (BUN; cat. no. SG7106B) and creatinine (Cre; GG7107A) ELISA kits (Beijing Wantai Drd Co., Ltd.) were used to determine the levels of Cre and BUN in the supernatant. In addition, the urine $(\sim 1 \mathrm{ml})$ from the rat cage was collected after $12 \mathrm{~h}$ of modeling and centrifuged at $1,000 \mathrm{x} \mathrm{g}$ at $4^{\circ} \mathrm{C}$ for $15 \mathrm{~min}$. The supernatant was collected, and the levels of kidney injury molecule 1 (KIM1, PK683) and neutrophil gelatinase-associated lipocalin (NGAL, PN758) in the urine were detected via ELISA kits (Beyotime Institute of Biotechnology) according to the manufacturer's instructions.

Detection of inflammatory factors. TNF- $\alpha$ (PT512), IL-6 (cat. no. PI326) and IL-1 $\beta$ (PI301) levels in the serum of 
A

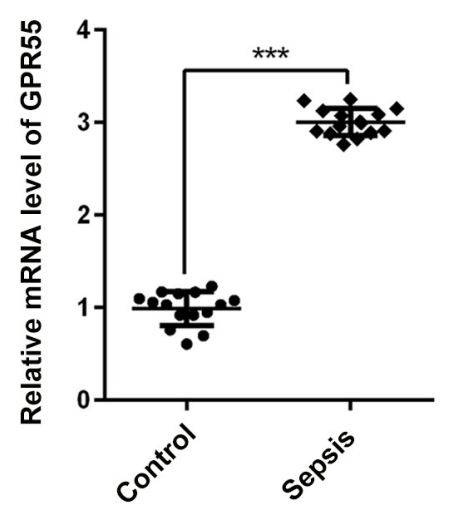

B

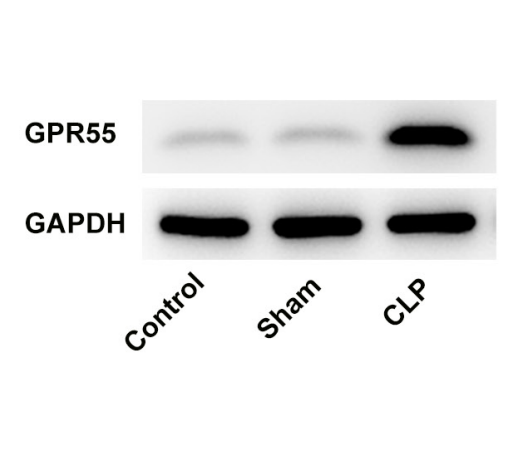

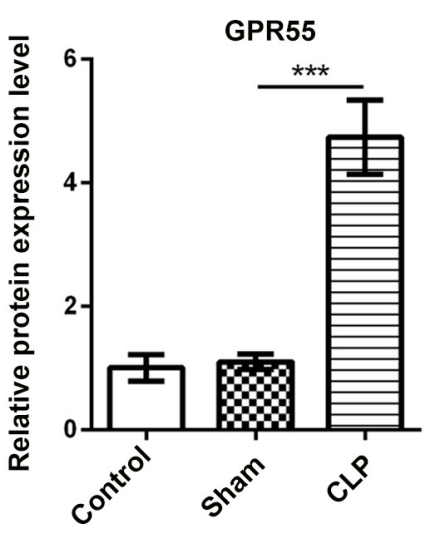

C

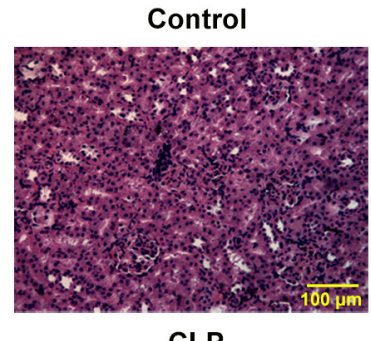

H\&E

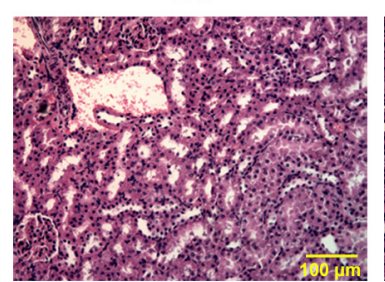

Sham

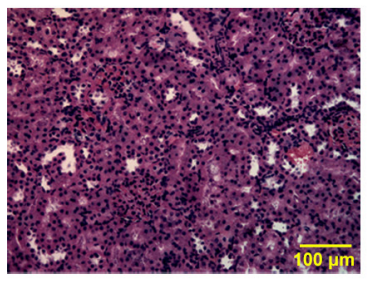

CID16020046

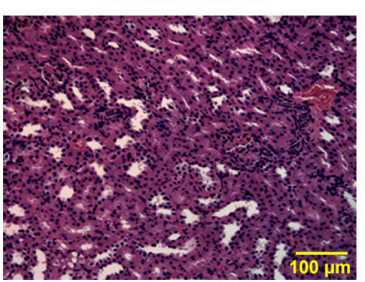

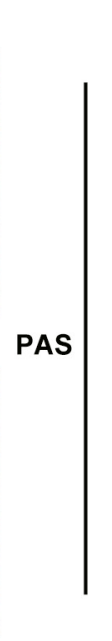

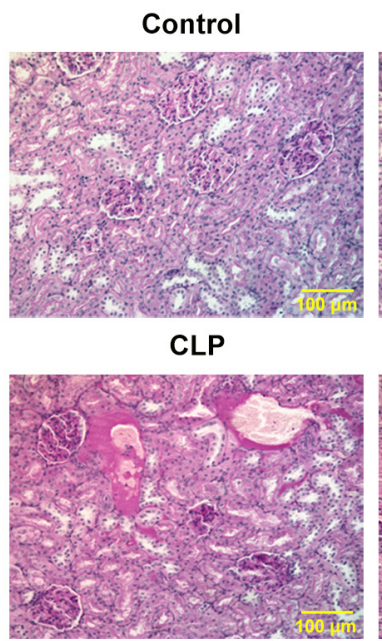

Sham
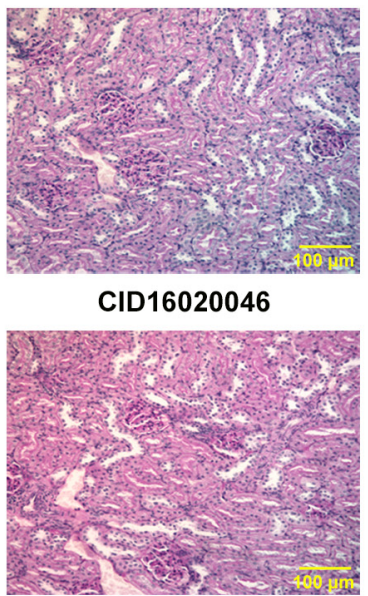

Figure 1. CID16020046 attenuates sepsis-induced renal injury. (A) Expression level of GPR55 in the serum of patients with sepsis was detected via reverse transcription-quantitative PCR. (B) Expression level of GPR55 in the renal tissue of septic mice was determined via western blotting. (C) Renal histopathology was assessed by PAS and H\&E staining. Magnification, $\mathrm{x} 200{ }^{*}{ }^{* * *} \mathrm{P}<0.001$. PAS, periodic acid-Schiff; GPR55, G protein-coupled receptor 55; CLP, cecal ligation/perforation.

septic mice were detected using respective ELISA kits according to the manufacturer's instructions (Beyotime Institute of Biotechnology). In brief, $2 \mathrm{ml}$ blood was collected from mice at room temperature and centrifuged at $4{ }^{\circ} \mathrm{C}$ for $5 \mathrm{~min}$ at 3,000 $\mathrm{x}$. The absorbance of the serum sample was measured at $450 \mathrm{~nm}$ using an Automatic Microplate reader (Syngene).

TUNEL staining assay. The TUNEL Apoptosis Assay kit (cat. no. C1088; Beyotime Institute of Biotechnology) was used to detect the apoptosis of renal tissue of mice. Briefly, the paraffin-embedded tissues were deparaffinized with xylene and rehydrated with graded ethanol at room temperature. The renal tissue sections were washed with PBS, fixed with $1 \%$ paraformaldehyde at room temperature for $15 \mathrm{~min}$ and treated with proteinase $\mathrm{K}$ working solution for $10 \mathrm{~min}$ at $37^{\circ} \mathrm{C}$, followed by incubation with the TUNEL reaction mixture at $37^{\circ} \mathrm{C}$ for $60 \mathrm{~min}$ in the dark. The nucleus was stained with DAPI for $5 \mathrm{~min}$ at room temperature and mounted in an anti-fade reagent (Beijing Solarbio Science \& Technology Co., Ltd.). In total, five random fields (magnification, x200) were imaged using an inverted fluorescence microscope (Olympus IX71; Olympus Corporation).
Western blot analysis. Total proteins were extracted from cells and renal tissues using RIPA lysis buffer containing protease inhibitors (Beyotime Institute of Biotechnology). The protein concentration was detected using a BCA assay kit (cat. no. BCA-02; Beijing Dingguo Changsheng Biotechnology Co., Ltd.). The proteins $(30 \mu \mathrm{g})$ were separated by $12 \%$ SDS-PAGE and transferred to PVDF membranes. Next, these membranes were blocked with $5 \%$ skimmed milk powder for $2 \mathrm{~h}$ at room temperature, and then incubated overnight at $4^{\circ} \mathrm{C}$ with the primary antibodies (diluted 1:1,000) against GPR55 (cat. no. ab203663; Abcam), TNF- $\alpha$ (cat. no. ab215188; Abcam), IL-6 (cat. no. ab233706; Abcam), IL-1 $\beta$ (cat. no. ab234437; Abcam), Bax (cat. no. ab32503; Abcam), Bcl-2 (cat. no. ab182858; Abcam), caspase3 (cat. no. ab13847; Abcam), cleaved caspase3 (cat. no. ab2302; Abcam), caspase9 (cat. no. ab32539; Abcam), cleaved caspase9 (cat. no. ab2324; Abcam), Ras homolog family member A (RhOA; cat. no. ab187027; Abcam), Rho-associated protein kinase (ROCK) 1 (cat. no. ab134181; Abcam), ROCK2 (cat. no. ab125025; Abcam) and GAPDH (cat. no. ab9485; Abcam). Subsequently, the membranes were washed with TBS- $0.1 \%$ Tween 20 and then co-incubated with secondary antibodies conjugated to $\operatorname{HRP}(1: 5,000$; cat. no. sc-2357, Santa Cruz Biotechnology, Inc.) for $2 \mathrm{~h}$ at room temperature. The 

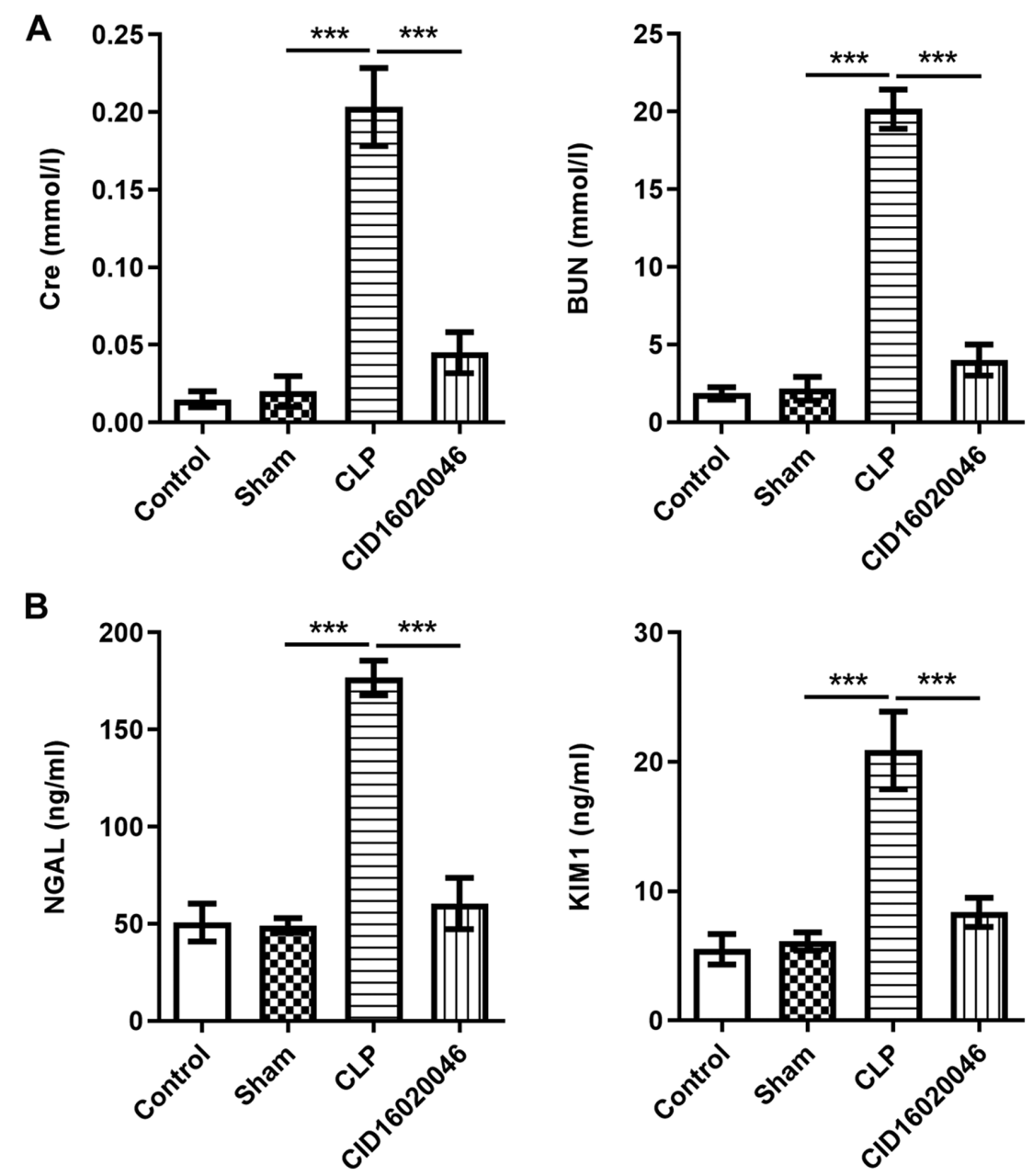

Figure 2. CID16020046 decreases the level of renal injury biomarkers induced by sepsis. (A) Effect of CID16020046 on the levels of Cre and BUN in the serum of septic mice was measured via ELISA. (B) Effect of CID16020046 on the levels of KIM1 and NGAL in the urine of septic mice was measured via ELISA. ${ }^{* * * *} \mathrm{P}<0.001$. CLP, cecal ligation/perforation; Cre, creatinine; BUN, blood urea nitrogen; KIM1, kidney injury molecule-1; NGAL, neutrophil gelatinase-associated lipocalin.

protein bands were detected using an ECL kit (Amersham Biosciences; Cytiva) and analyzed with ImageJ version 1.46 software (National Institutes of Health). GAPDH was used as an internal reference.

Statistical analysis. All data were analyzed using GraphPad Prism Software 8 (GraphPad Software, Inc.). All experimental data are expressed as the mean \pm SEM, and each experiment was repeated $\geq 3$ times. An unpaired Student's t-test was performed to compare two groups, and one-way ANOVA followed by Tukey's post hoc test was used for the comparison among multiple groups. For clinical analysis, the expression level of GRP55 between patients and healthy controls was analyzed using the Wilcoxon-Mann-Whitney test. $\mathrm{P}<0.05$ was considered to indicate statistically significant differences.

\section{Results}

CID16020046 attenuates sepsis-induced renal injury. In order to examine the effect of GPR55 on sepsis-induced renal injury, the expression level of GPR55 was first determined in the serum of healthy subjects and patients with sepsis. As shown in Fig. 1A, the results of RT-qPCR revealed that the expression level of GPR55 in the serum of patients with sepsis was significantly higher compared with that in the control group. Next, the role of GPR55 in sepsis-induced renal injury was further evaluated using CLP to construct a murine model of polymicrobial sepsis. As presented in Fig. 1B, the expression level of GPR55 in the CLP group was significantly higher compared with that in the sham group.

Renal pathological injury was analyzed via H\&E and PAS staining. The results of H\&E and PAS staining demonstrated that the complete renal glomerulus and tubule morphology was destroyed after CLP operation, indicating a more prominent renal injury in the CLP group compared with that in the sham group, whereas the GPR55 inhibitor CID16020046 (20 mg/kg) notably decreased the thickening of the basement membrane of renal tubules and glomerular hypertrophy, thereby alleviating the severity of renal pathological injury induced by sepsis (Fig. 1C).

CID16020046 reduces the levels of renal injury biomarkers induced by sepsis. To further verify the protective effect of CID16020046 against sepsis-induced renal injury, the levels of Cre and BUN in the serum, and the levels of KIM1 and NGAL in the urine of septic mice were measured by ELISA. 
A

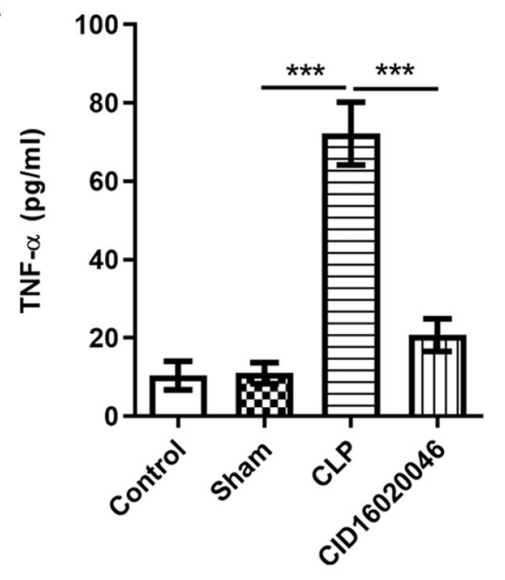

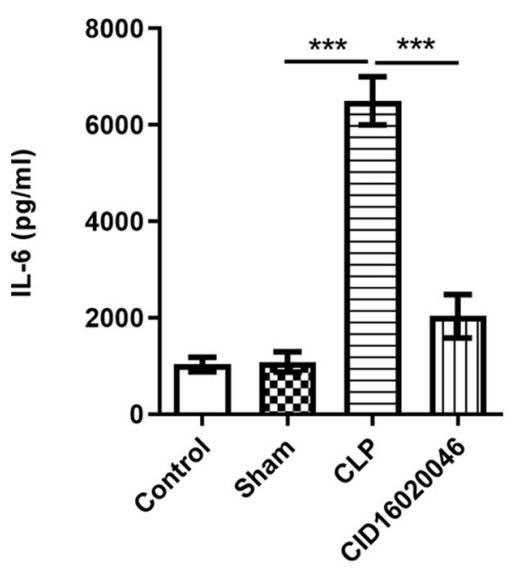

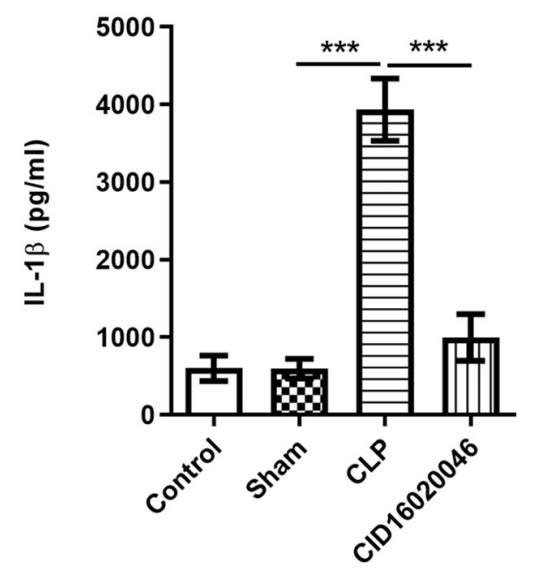

B
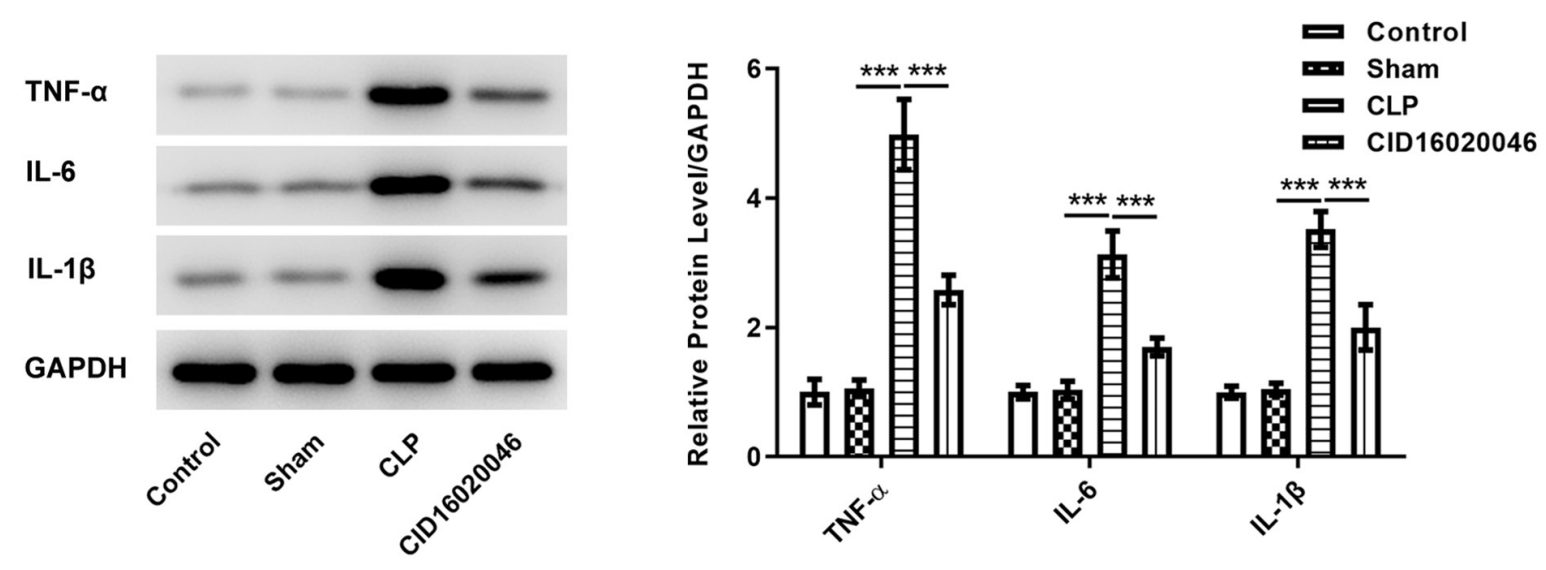

Figure 3. CID16020046 alleviates inflammation in serum and renal tissue induced by sepsis. (A) Effect of CID16020046 on the levels of the pro-inflammatory cytokines, TNF- $\alpha$, IL-6 and IL-1 $\beta$, in the serum of septic mice was detected via ELISA. (B) Effect of CID16020046 on the levels of pro-inflammatory cytokines (TNF- $\alpha$, IL-6 and IL-1 $\beta$ ) in the renal tissue of septic mice was detected via ELISA. ${ }^{* * *} \mathrm{P}<0.001$. CLP, cecal ligation/perforation.

As presented in Fig. 2A, the levels of Cre and BUN in the CLP group were significantly higher compared with those in the sham group. Moreover, the levels of KIM1 and NGAL in the CLP group were significantly increased compared with those in the sham group (Fig. 2B). However, these elevated levels of Cre, BUN, KIM1 and NGAL in mice receiving CLP operation were significantly reduced after the administration of CID16020046 $(20 \mathrm{mg} / \mathrm{kg})$. These results indicated that CID16020046 could effectively improve renal tissue injury induced by sepsis.

CID16020046 alleviates inflammation in the serum and renal tissue induced by sepsis. ELISA and western blotting were performed to examine the sepsis-induced levels of the pro-inflammatory factors TNF- $\alpha$, IL- 6 and IL- $1 \beta$. ELISA results demonstrated that the levels of TNF- $\alpha$, IL- 6 and IL- $1 \beta$ were significantly increased in the CLP group compared with those in the sham group, while CID16020046 effectively suppressed the levels of TNF- $\alpha$, IL-6 and IL-1 $\beta$ (Fig. 3A). The western blotting results also identified that CID16020046 partially abolished the upregulation of TNF- $\alpha$, IL- 6 and IL-1 $\beta$ expression induced by sepsis (Fig. 3B), suggesting that CID16020046 alleviates inflammation in sepsis-induced AKI.

CID16020046 reduces renal cell apoptosis induced by sepsis. Cell apoptosis serves an important role in the occurrence and development of sepsis (14). Next, a TUNEL staining assay and western blotting were used to determine the extent of cell apoptosis in renal tissue. As shown in Fig. 4A, the results of TUNEL staining demonstrated that the fluorescence intensity of renal tissue cells in the CID16020046 group was weaker compared with that in the CLP group, indicating that CID16020046 markedly alleviated the apoptosis of renal tissue cells induced by sepsis. In addition, the western blotting results revealed that, compared with the CLP group, CID16020046 significantly inhibited the downregulation of the anti-apoptotic protein Bcl-2 and the upregulation of the pro-apoptotic proteins Bax, cleaved caspase 3 and cleaved caspase9 induced by sepsis (Fig. 4B).

CID16020046 ameliorates sepsis-induced renal injury by inhibiting the RhOA/ROCK pathway. The effect of GPR55 on the expression levels of RhOA/ROCK pathway-related proteins were determined via western blotting. As shown in Fig. 5, the expression levels of RhOA, ROCK1 and ROCK2 in the CLP group were significantly higher compared with those in the sham group, whereas CD16020046 significantly reduced the increased expression levels of RhOA, ROCK 1 and ROCK 2 induced by sepsis. These results indicated that CID16020046 could effectively alleviate sepsis-induced renal injury by suppressing the RhOA/ROCK pathway. 
A

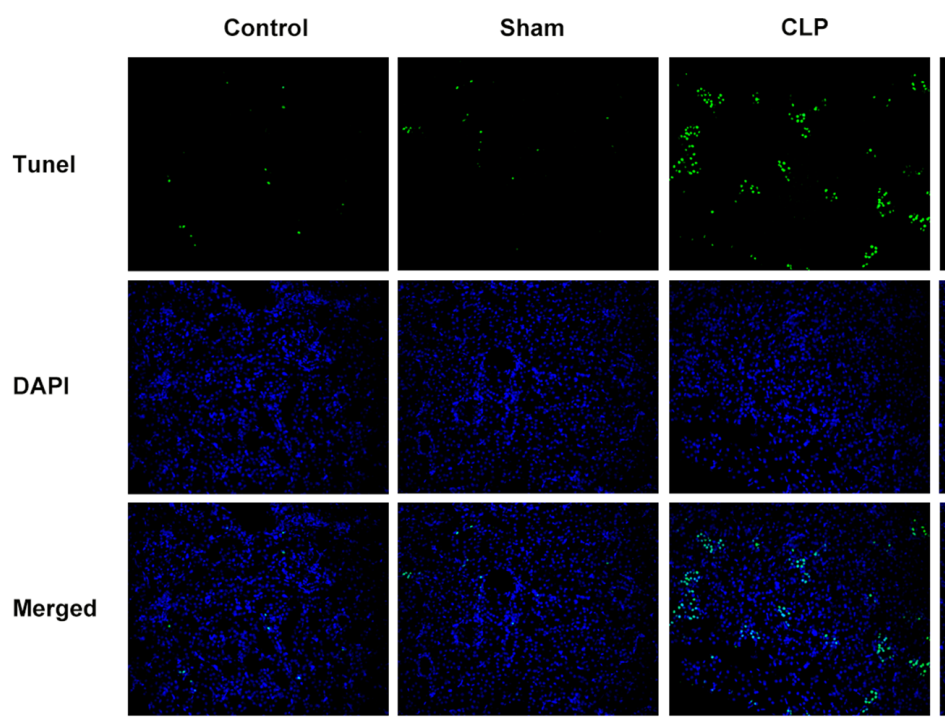

B

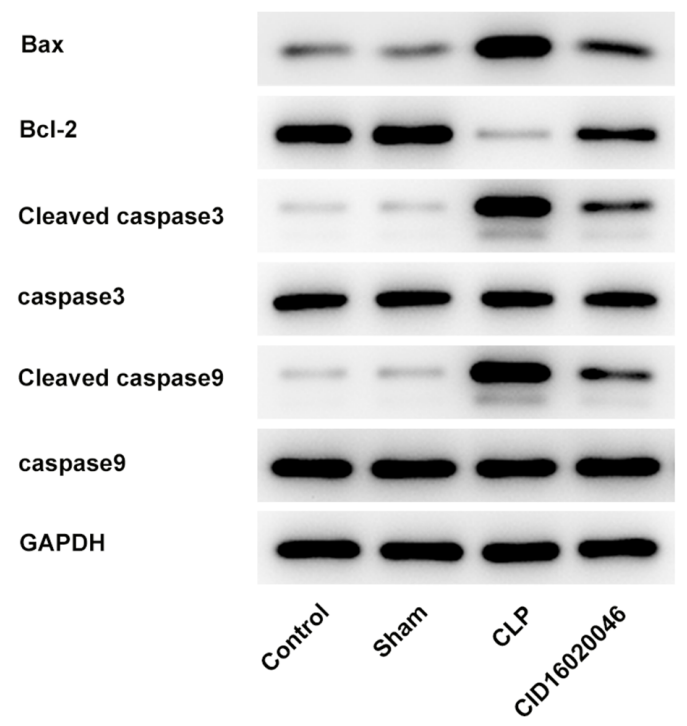

CID16020046

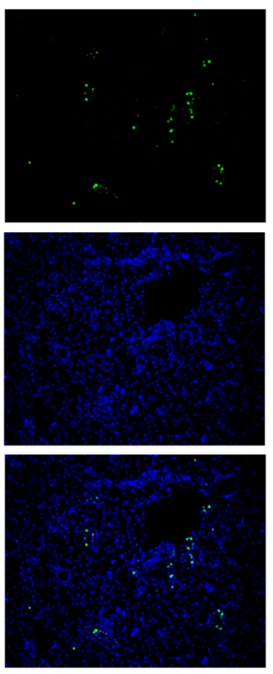

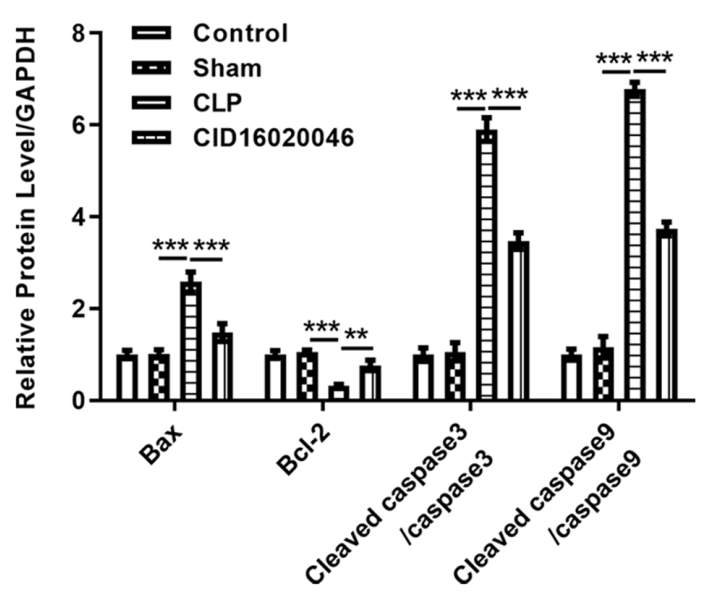

Figure 4. CID16020046 reduces the cell apoptosis in renal tissue induced by sepsis. (A) Effect of CID16020046 on the apoptosis in the cells of kidney tissue induced by sepsis was determined via TUNEL assay. Scale bar, $100 \mu \mathrm{m}$. (B) Effect of CID16020046 on the apoptosis-associated proteins (Bax, Bcl-2, caspase3, cleaved caspase3, cleaved caspase9 and caspase9) in the cells of kidney tissue induced by sepsis was analyzed via western blotting. Magnification, $\mathrm{x} 200$. ${ }^{* *} \mathrm{P}<0.01$ and ${ }^{* * *} \mathrm{P}<0.001$. CLP, cecal ligation/perforation.

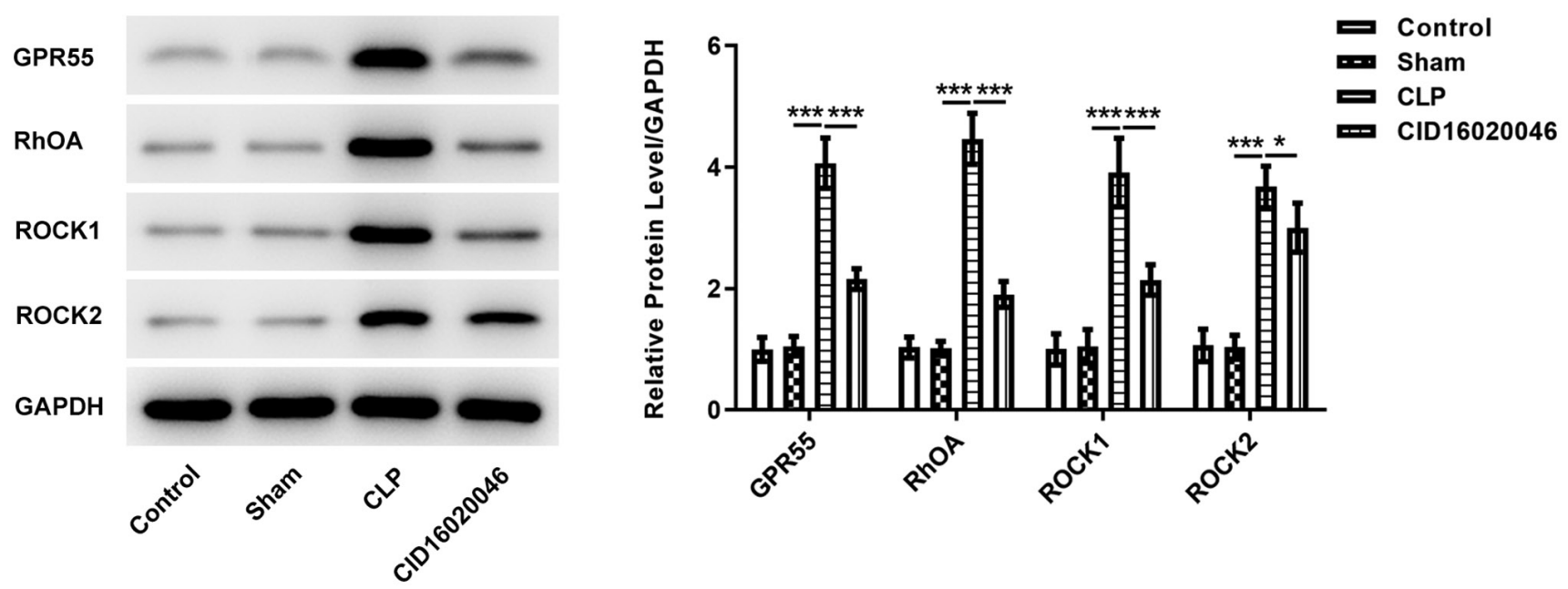

Figure 5. CID16020046 ameliorates sepsis-induced renal injury by inhibiting the RhOA/ROCK pathway. The protein expression levels of GRP55, RhOA, ROCK1 and ROCK2 in kidney tissues of in different groups were detected via western blotting. ${ }^{*}<0.05$ and ${ }^{* * * *} \mathrm{P}<0.001$. CLP, cecal ligation/perforation; RhOA, ras homolog family member A; ROCK, Rho-associated protein kinase. 

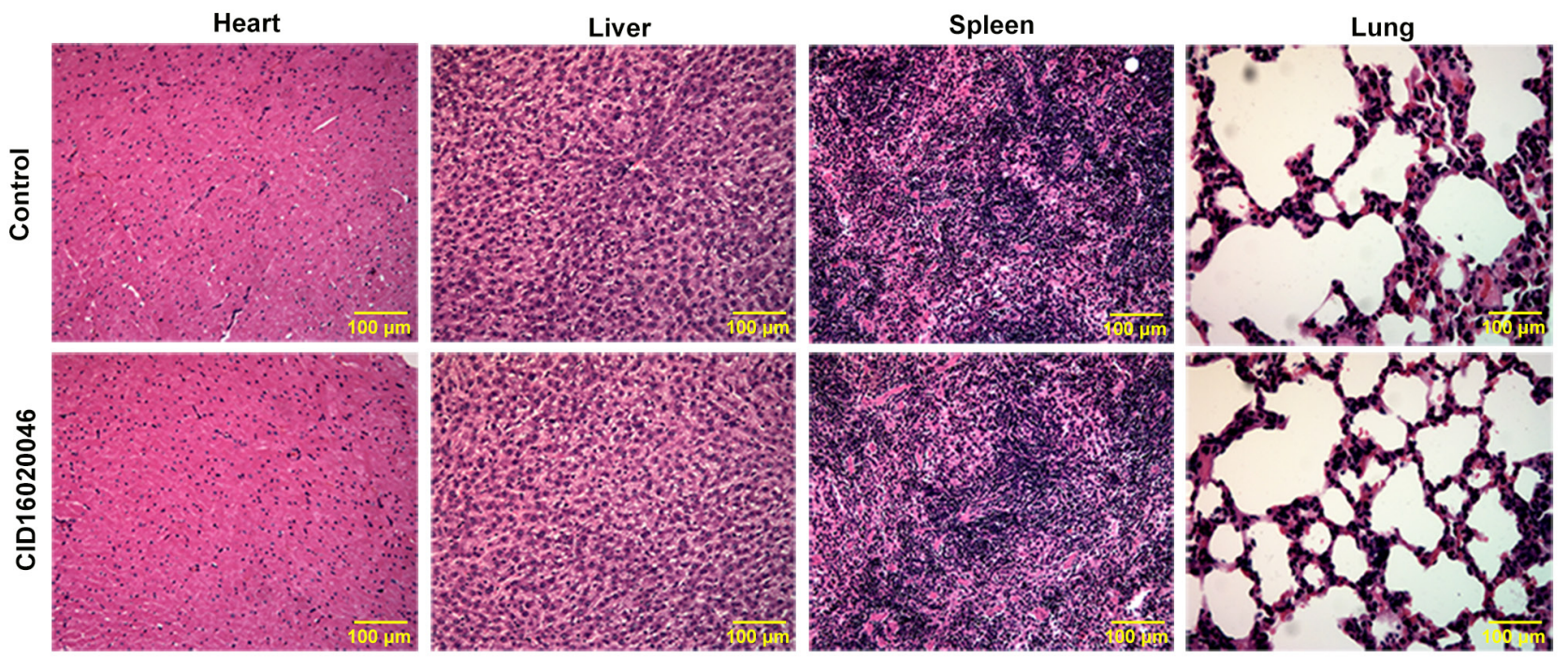

Figure 6. CID16020046 does not adversely affect the heart, liver, spleen or lung. The histological images of heart, liver, spleen and lung in normal mice with or without treatment with CID16020046 $(20 \mathrm{mg} / \mathrm{kg})$ were analyzed by H\&E staining. Magnification, x200.

CID16020046 does not adversely affect the heart, liver, spleen or lung. Finally, the toxicity of CID16020046 $(20 \mathrm{mg} / \mathrm{kg})$ on the heart, liver, spleen and lung in normal mice was analyzed by H\&E staining. The results demonstrated that there were no apparent pathological changes in the heart, liver, spleen or lung between normal mice treated with $20 \mathrm{mg} / \mathrm{kg}$ CID16020046 or normal mice without treatment (Fig. 6).

\section{Discussion}

In the present study, a CLP-induced sepsis mouse model was constructed to analyze the effect of CID16020046 on renal injury induced by sepsis. The results demonstrated that GPR55 expression was upregulated in the renal tissue of septic mice. It was found that the GPR55 inhibitor CID16020046 could improve the sepsis-induced renal injury, including inflammation and apoptosis, by inhibiting the activation of the RhOA/ROCK signaling pathway. In addition, CID16020046 at $20 \mathrm{mg} / \mathrm{kg}$ was proven to exert no toxic side effects in normal mice.

As it was determined in a previous study that inhibition of GPR55 could attenuate experimental sepsis, the role of GPR55 in sepsis-induced AKI was further investigated. Cre and BUN levels in the serum mainly reflect glomerular filtration function, and so, these two indices are often used to determine the integrity of renal function $(15,16)$. KIM1 is a transmembrane glycoprotein that can be cleaved into soluble fragments and is eventually excreted into urine to participate in the process of immune tolerance $(17,18)$. Moreover, NGAL is a type of injury-induced transferrin that may improve the activation of intracellular coenzyme iron and regulate various important proteins involved in cellular activities (19). For example, Guo et al (20) revealed that NGAL modulates cell apoptosis and epithelial-mesenchymal transition in nasopharyngeal carcinoma. Han et al (21) demonstrated that NGAL participates in LPS-mediated apoptosis of renal tubular epithelial cells. Therefore, KIM1 and NGAL are often used as markers of acute renal tubular injury. In the present study, GPR55 expression was upregulated in the serum of patients with sepsis and in the renal tissue of mice with CLP-induced sepsis. Thus, CID16020046 was applied to reduce GPR55, and the results demonstrated that CID16020046 significantly lowered the level renal injury biomarkers (Cre, BUN, KIM1 and NGAL), directly suggesting that CID16020046 possessed a potential protective function in sepsis-induced AKI.

Subsequently, how CID16020046 exerted its protective effects on sepsis-induced AKI was further evaluated. Previous studies have reported that the GPR55 antagonist CID16020046 exhibited notable anti-inflammatory properties in different disease processes. For example, CID16020046 could effectively suppress intestinal inflammation and endothelial cell inflammation induced by the oxidized low-density lipoprotein $(10,22)$. These studies indicated that CID16020046 exerted notable anti-inflammatory effects and may hold promise in clinical application. In agreement with these previous reports, CID16020046 also exhibited significant inhibitory effects on the production of TNF- $\alpha$, IL- 6 and IL- $1 \beta$ both in serum and kidney tissues in septic mice in the present study. Therefore, it was indicated that CID16020046 may have a positive protective effect against sepsis-induced renal injury by inhibiting inflammation.

In addition to inflammation, tubular cell apoptosis has been recognized as another main histopathologic characteristic in sepsis-induced AKI (23). A recent study revealed that GPR55 was involved in apoptosis processing (24). The antagonists of GPR 55 were able to block apoptosis in PC12 cells, whereas GPR55 agonists protected against endoplasmic reticulum-induced apoptosis in pancreatic $\beta$ cells $(25,26)$. In the present study, apoptotic cells were more likely to occur in the septic mice, accompanied with the highly expressed pro-apoptotic proteins and the lowly expressed anti-apoptotic proteins. The administration of CID16020046 markedly attenuated this condition, suggesting that CID16020046 may have a positive protective effect against sepsis-induced renal injury by inhibiting apoptosis. 
To further study the effect of CID16020046 on renal injury induced by sepsis, its effect on the potential downstream regulatory pathway RhOA/ROCK was investigated. It was previously reported that RhOA may be the molecular initiator of intracellular signal transduction (27). ROCK, a downstream protein of RhOA kinase, can phosphorylate a variety of substrates, and is closely associated with tumor invasion and metastasis, tissue and organ fibrosis, nerve regeneration and remodeling, and the occurrence and development of cardio-cerebrovascular diseases (28). In addition, ROCK serves a key role in inflammation and autoimmune diseases. For example, a previous study revealed that ROCK may participate in the activation of the NF- $\kappa \mathrm{B}$ pathway and induce the production of TNF- $\alpha$ and other inflammatory factors (29). In addition, ROCK inhibitors were found to attenuate the nephrotoxicity of chemicals, inhibit inflammatory and apoptotic factors, and reduce renal fibrosis $(30,31)$. Of note, inhibition of the RhOA/ROCK signaling pathway markedly decreases sepsis-induced AKI in rats (32). Furthermore, GPR55 can directly regulate the expression levels of downstream effectors of ROCK signaling $(33,34)$. Therefore, it may be hypothesized that CID16020046 improves sepsis-induced renal injury by inhibiting ROCK signaling. In the present study, CID16020046 significantly inhibited RhOA/ROCK signaling and participated in the regulation of renal injury induced by sepsis.

However, some limitations existed in the current study. This study preliminarily suggested that CID16020046 exerted effects in sepsis-induced AKI by regulating the RhOA/ROCK pathway, but an inhibition assay should be conducted to validate the protective mechanism mediated by the ROCK pathway. These experiments will be conducted in future work.

In conclusion, the GPR55 antagonist CID16020046 was shown to significantly reduce renal injury, including inflammation and apoptosis, induced by sepsis. Moreover, it was identified that the protective effect of CID16020046 may be mediated via inhibition of the RhOA/ROCK pathway. These results may provide the theoretical basis for the treatment of sepsis-induced AKI by CID16020046, but further research is required to fully elucidate the function and underlying mechanism of action of CID16020046.

\section{Acknowledgements}

Not applicable.

\section{Funding}

This study was supported by the Guangdong Provincial Natural Science Foundation (grant no. 2018A030313434), the Guangdong Provincial Medical Science Research Foundation (grant no. A2018259) and the Guangzhou Municipal Science and Technology Program (grant no. 201904010006).

\section{Availability of data and materials}

The datasets used and/or analyzed during the current study are available from the corresponding author on reasonable request.

\section{Authors' contributions}

RC and ZC designed the experimental study. RC, HX, ZG, PZ and $\mathrm{JC}$ performed the experiments, analyzed and interpreted the data. RC and HX wrote the manuscript. ZC revised the manuscript. All authors confirm the authenticity of all the raw data. All authors have read and approved the final version of the manuscript.

\section{Ethics approval and consent to participate}

This present study was performed based on the principles expressed in the Declaration of Helsinki. The present study was approved by the Ethics Committee of The Second Affiliated Hospital of Guangzhou Medical University and conducted in accordance with the National Institutes of Health Guide for the Care and Use of Laboratory Animals. Each participant provided signed informed consent prior to participate in the present study. Patients or their legal surrogates provided signed informed consent for the surgical procedures.

The animal experimental procedures were approved by the Animal Committee of The Second Affiliated Hospital of Guangzhou Medical University.

\section{Patient consent for publication}

Not applicable.

\section{Competing interests}

The authors declare that they have no competing interests.

\section{References}

1. Fleischmann C, Scherag A, Adhikari NK, Hartog CS, Tsaganos T, Schlattmann P, Angus DC and Reinhart K; International Forum of Acute Care Trialists: Assessment of global incidence and mortality of hospital-treated sepsis. Current estimates and limitations. Am J Respir Crit Care Med 193: 259-272, 2016

2. Shankar-Hari M, Phillips GS, Levy ML, Seymour CW, Liu VX, Deutschman CS, Angus DC, Rubenfeld GD and Singer M; Sepsis Definitions Task Force: Developing a new definition and assessing new clinical criteria for septic shock: For the third international consensus definitions for sepsis and septic shock (sepsis-3). JAMA 315: 775-787, 2016.

3. van der Poll T, van de Veerdonk FL, Scicluna BP and Netea MG: The immunopathology of sepsis and potential therapeutic targets. Nat Rev Immunol 17: 407-420, 2017.

4. Vincent JL, Jones G, David S, Olariu E and Cadwell KK: Frequency and mortality of septic shock in Europe and North America: A systematic review and meta-analysis. Crit Care 23: 196, 2019.

5. Uchino S, Kellum JA, Bellomo R, Doig GS, Morimatsu H, Morgera S, Schetz M, Tan I, Bouman C, Macedo E, et al: Acute renal failure in critically ill patients: A multinational, multicenter study. JAMA 294: 813-818, 2005.

6. Ma S, Evans RG, Iguchi N, Tare M, Parkington HC, Bellomo R, May CN and Lankadeva YR: Sepsis-induced acute kidney injury: A disease of the microcirculation. Microcirculation 26: e12483, 2019.

7. Sharir $\mathrm{H}$ and Abood ME: Pharmacological characterization of GPR55, a putative cannabinoid receptor. Pharmacol Ther 126: 301-313, 2010.

8. Schicho R, Bashashati M, Bawa M, McHugh D, Saur D, Hu HM, Zimmer A, Lutz B, Mackie K, Bradshaw HB, et al: The atypical cannabinoid $\mathrm{O}-1602$ protects against experimental colitis and inhibits neutrophil recruitment. Inflamm Bowel Dis 17: 1651-1664, 2011. 
9. Staton PC, Hatcher JP, Walker DJ, Morrison AD, Shapland EM, Hughes JP, Chong E, Mander PK, Green PJ, Billinton A, et al: The putative cannabinoid receptor GPR55 plays a role in mechanical hyperalgesia associated with inflammatory and neuropathic pain. Pain 139: 225-236, 2008

10. Stančić A, Jandl K, Hasenöhrl C, Reichmann F, Marsche G, Schuligoi R, Heinemann A, Storr M and Schicho R: The GPR55 antagonist CID16020046 protects against intestinal inflammation. Neurogastroenterol Motil 27: 1432-1445, 2015.

11. Zhou J, Yang H and Lehmann C: Inhibition of GPR 55 improves dysregulated immune response in experimental sepsis. Clin Hemorheol Microcirc 70: 553-561, 2018.

12. Zeng Q, Wu J and Yang S: Circulating lncRNA ITSN1-2 is upregulated, and its high expression correlates with increased disease severity, elevated inflammation, and poor survival in sepsis patients. J Clin Lab Anal 33: e22836, 2019.

13. Livak KJ and Schmittgen TD: Analysis of relative gene expression data using real-time quantitative PCR and the 2(-Delta Delta C(T)) method. Methods 25: 402-408, 2001.

14. Harjai M, Bogra J, Kohli M and Pant AB: Is suppression of apoptosis a new therapeutic target in sepsis? Anaesth Intensive Care 41: 175-183, 2013

15. Song S, Meyer M, Türk TR, Wilde B, Feldkamp T, Assert R, Wu K, Kribben A and Witzke O: Serum cystatin C in mouse models: A reliable and precise marker for renal function and superior to serum creatinine. Nephrol Dial Transplant 24: 1157-1161, 2009.

16. Rittirsch D, Huber-Lang MS, Flierl MA and Ward PA Immunodesign of experimental sepsis by cecal ligation and puncture. Nat Protoc 4: 31-36, 2009.

17. Han WK, Waikar SS, Johnson A, Betensky RA, Dent CL, Devarajan $\mathrm{P}$ and Bonventre JV: Urinary biomarkers in the early diagnosis of acute kidney injury. Kidney Int 73: 863-869, 2008.

18. Kuchroo VK, Umetsu DT, DeKruyff RH and Freeman GJ: The TIM gene family: Emerging roles in immunity and disease. Nat Rev Immunol 3: 454-462, 2003.

19. Kuwabara T, Mori K, Mukoyama M, Kasahara M, Yokoi H, Saito Y, Yoshioka T, Ogawa Y, Imamaki H, Kusakabe T, et al: Urinary neutrophil gelatinase-associated lipocalin levels reflect damage to glomeruli, proximal tubules, and distal nephrons. Kidney Int 75: 285-294, 2009.

20. Guo Y, Zhai J, Zhang J and Zhou H: NGAL protects in nasopharyngeal carcinoma by inducing apoptosis and blocking epithelial-mesenchymal transition. Oncol Lett 19: 3711-3718, 2020.

21. Han M, Pan Y, Gao M, Zhang J and Wang F: JNK signaling pathway suppresses LPS-mediated apoptosis of HK-2 cells by upregulating NGAL. Int J Inflam 2020: 3980507, 2020.

22. Wang Y, Pan W, Wang Y and Yin Y: The GPR55 antagonist CID16020046 protects against ox-LDL-induced inflammation in human aortic endothelial cells (HAECs). Arch Biochem Biophys 681: 108254, 2020.
23. Garofalo AM, Lorente-Ros M, Goncalvez G, Carriedo D, Ballén-Barragán A, Villar-Fernández A, Peñuelas Ó, Herrero R, Granados-Carreño R and Lorente JA: Histopathological changes of organ dysfunction in sepsis. Intensive Care Med Exp 7 (Suppl 1): S45, 2019.

24. Vong CT, Tseng HHL, Kwan YW, Lee SM and Hoi MPM: Novel protective effect of O-1602 and abnormal cannabidiol, GPR55 agonists, on ER stress-induced apoptosis in pancreatic $\beta$-cells. Biomed Pharmacother 111: 1176-1186, 2019.

25. Akimov MG, Ashba AM, Gretskaya NM and Bezuglov VV: N-acyl dopamines induce apoptosis in PC12 cell line via the GPR55 receptor activation. Dokl Biochem Biophys 474: 155-158, 2017.

26. Vong CT, Tseng HHL, Kwan YW, Lee SM and Hoi MPM: Novel protective effect of O-1602 and abnormal cannabidiol, GPR55 agonists, on ER stress-induced apoptosis in pancreatic $\beta$-cells. Biomed Pharmacother 111: 1176-1186, 2019.

27. Wan L, Bagshaw SM, Langenberg C, Saotome T, May C and Bellomo R: Pathophysiology of septic acute kidney injury: What do we really know? Crit Care Med 36 (4 Suppl): S198-S203, 2008.

28. Lochhead PA, Wickman G, Mezna M and Olson MF: Activating ROCK1 somatic mutations in human cancer. Oncogene 29: 2591-2598, 2010

29. Shimizu S, Tahara M, Ogata S, Hashimoto K, Morishige K, Tasaka K and Murata Y: Involvement of nuclear factor-kB activation through RhoA/Rho-kinase pathway in LPS-induced IL-8 production in human cervical stromal cells. Mol Hum Reprod 13: 181-187, 2007.

30. Park JW, Park CH, Kim IJ, Bae EH, Ma SK, Lee JU and Kim SW: Rho kinase inhibition by fasudil attenuates cyclosporine-induced kidney injury. J Pharmacol Exp Ther 338: 271-279, 2011.

31. Hu H, Chen W, Ding J, Jia M, Yin J and Guo Z: Fasudil prevents calcium oxalate crystal deposit and renal fibrogenesis in glyoxylate-induced nephrolithic mice. Exp Mol Pathol 98: 277-285, 2015.

32. Yan XX, Zheng AD, Zhang ZE, Pan GC and Zhou W: Protective effect of pantoprazole against sepsis-induced acute lung and kidney injury in rats. Am J Transl Res 11: 5197-5211, 2019.

33. Vong CT, Tseng HHL, Kwan YW, Lee SM and Hoi MPM: G-protein coupled receptor 55 agonists increase insulin secretion through inositol trisphosphate-mediated calcium release in pancreatic $\beta$-cells. Eur J Pharmacol 854: 372-379, 2019.

34. Robertson-Gray OJ, Walsh SK, Ryberg E, Jönsson-Rylander AC, Lipina $C$ and Wainwright CL: 1- $\alpha$-Lysophosphatidylinositol (LPI) aggravates myocardial ischemia/reperfusion injury via a GPR55/ROCK-dependent pathway. Pharmacol Res Perspect 7: e00487, 2019.

This work is licensed under a Creative Commons Attribution-NonCommercial-NoDerivatives 4.0 International (CC BY-NC-ND 4.0) License. 